\title{
Magnetic Properties and Magnetoimpedace of Electroplated Wires
}

\author{
G.V. Kurlyandskaya ${ }^{1,2, a, *}$, P. Jantaratana ${ }^{3, b}$, N.G. Bebenin ${ }^{4, c}$, \\ and V.O. Vas'kovskiy ${ }^{2, d}$ \\ ${ }^{1}$ University of the Basque Country UPV-EHU, Dept. Electricity and Electronics, Campus of Leioa, \\ 48940, Leioa, Spain \\ ${ }^{2}$ Ural State University, Dept. Magnetism and Magnetic Nanomaterials, Lenin Ave. 51, 620083, \\ Ekaterinburg, Russia \\ ${ }^{3}$ Kasetsart University, Dept of Physics, Bangkok 10900, Thailand \\ ${ }^{4}$ Institute of metal Physics, UD RAS, Kovalevskaya 18, 620990, Ekaterinburg, Russia \\ agalina@we.Ic.ehu.es, bfscipsj@ku.ac.th, ${ }^{\mathrm{c}}$ bebenin@imp.uran.ru, ${ }^{\mathrm{d}}$ vladimir.vaskovskiy@usu.ru, \\ ${ }^{*}$ corresponding author
}

Keywords: Magnetoimpedance, electroplated wires, magnetic anisotropy, magnetic field sensors.

\begin{abstract}
Magnetic properties and giant magnetoimpedance (GMI) of $\mathrm{CuBe} / \mathrm{Fe}_{20} \mathrm{Co}_{6} \mathrm{Ni}_{74}$ electroplated wires was studied for frequency of $1 \mathrm{MHz}$ and the amplitude of the driving current of $20 \mathrm{~mA}$ with focus on the development of magnetic field detectors of particular types. The possibility of quantitative ferrofluid concentration measurements was demonstrated. GMI of electroplated wire can be useful for the study of the dynamic properties of the ferrofluid. GMI of the electroplated wires in the non-uniform field created by the permanent magnet was studied in the configuration adapted for automatic control lines, machinery control, and positioning systems.
\end{abstract}

\section{Introduction}

The technology of preparation of composite wires with a thin magnetic electroplated coating was developed long ago being requested by applications of such materials in magnetic memory devices [1]. In 1996 Beach at el [2] has re-discovered these materials for technological applications (low magnetic field sensors) and basic studies of non-linear processes. Non-linearity of the magnetization processes was proved for different compositions of the electroplated [3-4] and composite [5] wires by Fourier analysis of the induced voltage (the high harmonics generation) and nonlinear dependence of the induced voltage on the driving current amplitude. It has been established that the high harmonics can show larger variation under application of an external magnetic field than the fundamental one. In order to explain the high sensitivity of the harmonics various theoretical models were proposed [2-4]. It was shown that for FeCoNi electroplated wires the strong nonlinear effects are the consequence of the high value of the transverse susceptibility at the points of re-orientational phase transitions in the magnetic layer and that the high-order magnetic anisotropy may play a key role [3-4]. Special attempts were made in order to design new family of the electroplated wires adapted for giant magnetoimpedance (GMI) applications [4, 6]. In this work, some recent results of the studies of physical processes in the electroplated wires with high GMI are discussed. Special attention is paid to some new applications of the GMI electroplated wires as sensitive elements of small magnetic field detectors of particular types.

\section{Experimental, results and discussion}

The $\mathrm{CuBe} / \mathrm{FeCoNi}$ a $100 \mu \mathrm{m}$ diameter composite wires were prepared by electrodeposition of $\mathrm{Fe}_{20} \mathrm{Co}_{6} \mathrm{Ni}_{74}$ magnetic layer of $1 \mu \mathrm{m}$ thick onto non-magnetic $\mathrm{CuBe}$ polished substrate. The length of the samples used for all measurements was $60 \mathrm{~mm}$. The longitudinal hysteresis loops of the samples were measured by conventional inductive technique. The observed values of the coercivity of 2 Oe and saturation field of about 8 Oe were similar to the values reported previously for 

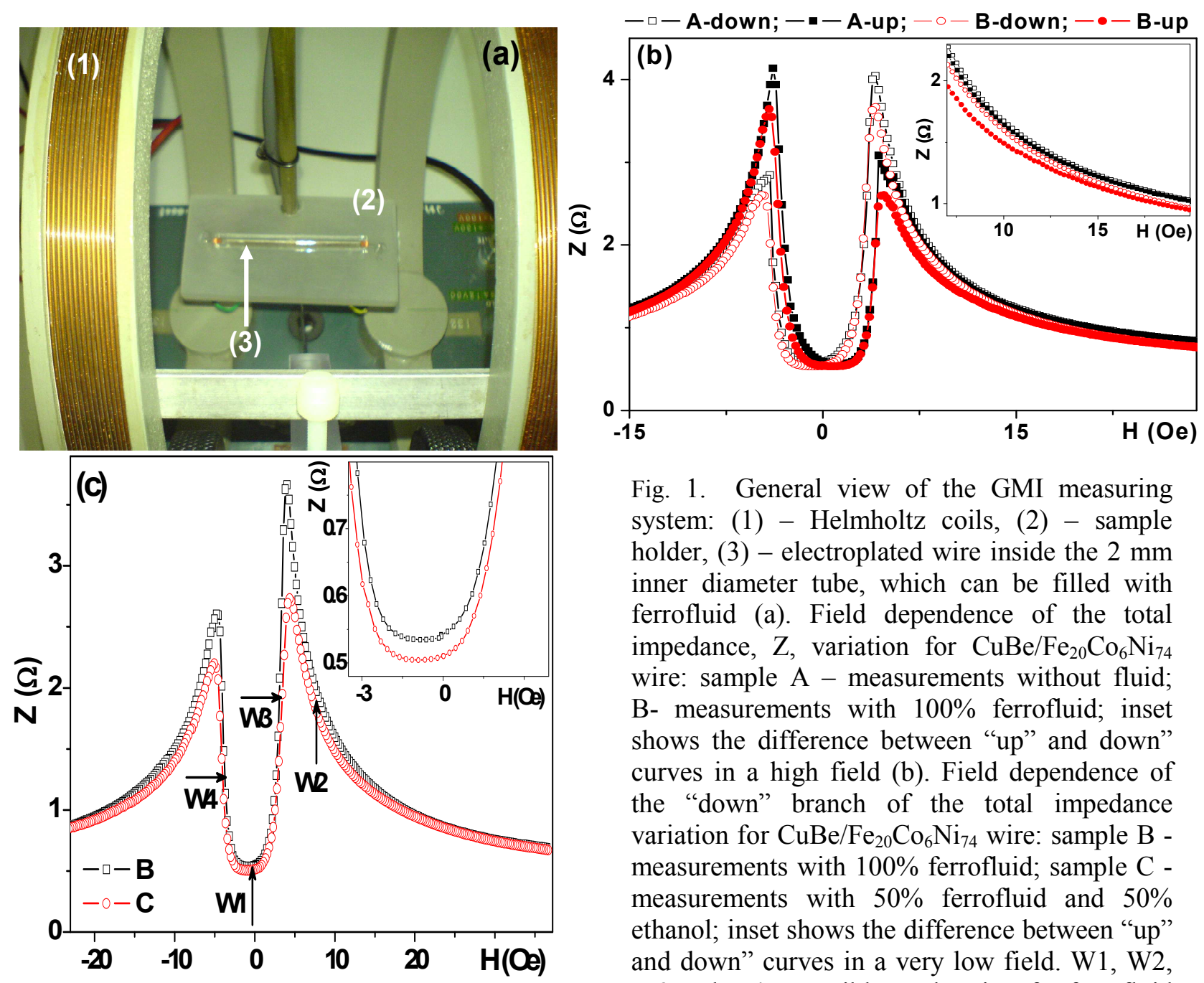

Fig. 1. General view of the GMI measuring system: (1) - Helmholtz coils, (2) - sample holder, (3) - electroplated wire inside the $2 \mathrm{~mm}$ inner diameter tube, which can be filled with ferrofluid (a). Field dependence of the total impedance, $\mathrm{Z}$, variation for $\mathrm{CuBe} / \mathrm{Fe}_{20} \mathrm{Co}_{6} \mathrm{Ni}_{74}$ wire: sample A - measurements without fluid; B- measurements with $100 \%$ ferrofluid; inset shows the difference between "up" and down" curves in a high field (b). Field dependence of the "down" branch of the total impedance variation for $\mathrm{CuBe} / \mathrm{Fe}_{20} \mathrm{Co}_{6} \mathrm{Ni}_{74}$ wire: sample $\mathrm{B}$ measurements with $100 \%$ ferrofluid; sample C measurements with $50 \%$ ferrofluid and $50 \%$ ethanol; inset shows the difference between "up" and down" curves in a very low field. W1, W2, W3 and W4 - possible work points for ferrofluid concentration measurements (c).

composite wires with close preparation parameters [3-4]. GMI was measured by a precision LCR meter (HP 4284A) for frequency (f) of $1 \mathrm{MHz}$ and the amplitude of the sinusoidal driving current of $20 \mathrm{~mA}$. GMI was measured both in increasing ("up") and decreasing external field ("down") curves. The GMI ratio was defined as $\Delta \mathrm{Z} / \mathrm{Z}(\mathrm{H})=100 \times\left[\mathrm{Z}(\mathrm{H})-\mathrm{Z}\left(\mathrm{H}_{\max }\right)\right] / \mathrm{Z}\left(\mathrm{H}_{\max }\right)$, where $\mathrm{H}_{\max }=38$ Oe . Very high GMI was observed at a frequency of $1 \mathrm{MHz}$, being of order of $440 \%$ for the first and of order of $280 \%$ for the second peak after saturation (Fig.1.). The difference between these peaks was previously discussed in terms of the orientation phase transitions of the first and the second order in the magnetic system with high order magnetic anisotropy [3-4]. One can select different working points for "down" branch of the impedance variation $(\mathrm{W} 1(\mathrm{H} \approx 0), \mathrm{W} 2(\mathrm{Z}(\mathrm{H} \approx+8 \mathrm{Oe})$, $\mathrm{W} 3(\mathrm{H}=+3.4 \mathrm{Oe})$ or $\mathrm{W} 4(\mathrm{H}=-4.0 \mathrm{Oe})$ as it is described in Figure 1 (c). The highest sensitivity with respect to an external field $(3.5 \Omega / \mathrm{Oe})$ and linear response corresponds to W3 work point. For W4 a linear response but lower sensitivity $(2.2 \Omega / \mathrm{Oe})$ and higher field interval were observed.

\section{GMI properties of the electroplated wires in presence of ferrofluids}

Magnetic properties of electroplated wires in presence of ferrofluid were recently studied by H.Garcia-Miquel et al [7]. They had analyzed the low frequency properties of $\mathrm{CuBe} / \mathrm{FeCoNi}$ composite wires with and without coating by ferrofluid and showed that low-frequency measurements for the frequencies up to $1 \mathrm{kHz}$ in presence of ferrofluid can be a useful method to study magnetic properties of ferromagnets. In this work GMI of $\mathrm{CuBe} / \mathrm{FeCoNi}$ wires was studied in presence of commercial FerroTec ${ }^{\circledR}$ ferrofluid [7] filled into the glass tube (Fig.1). The presence of 
the ferrofluid leads to the significant reduction of the GMI maximum and the increase of the GMI high field hysteresis (Fig. 1(b)). The changes in the shape of GMI curves are caused by both an increase of the dielectric permittivity, which is quite high for the water-based suspensions, and time dependent processes related to the increase of a magnetic damping. For example, in the presence of

\begin{tabular}{|c|c|c|}
\hline Sample & $\Delta \mathrm{Z} / \mathrm{Z}(\mathrm{H} 1), \%$ & $\Delta \mathrm{Z} / \mathrm{Z}(\mathrm{H} 2), \%$ \\
\hline A & 440 & 270 \\
\hline B & 430 & 275 \\
\hline C & 310 & 220 \\
\hline
\end{tabular}

Table 1. GMI values for "down" branch of the total impedance: sample A - measurements without fluid; B measurements with $100 \%$ ferrofluid; $\mathrm{C}$ - measurements with $50 \%$ ferrofluid and $50 \%$ ethanol. $\mathrm{H} 1$ - the field of the first and $\mathrm{H} 2$ of the second GMI maximum counted after the magnetic saturation.

$100 \%$ standard ferrofluid the difference between the GMI responses in increasing and decreasing magnetic field of $10 \mathrm{Oe}$ (Fig. 1 (b) ) was as high as $0.1 \mathrm{Ohm}$, i.e. five times higher than the difference between the GMI responses for the measurements without ferrofluid. Although there were successful examples of the detection of the presence of $100 \%$ standard ferrofluid spread on the surface of the magnetic material by GMI response change [8], the possibility of the evaluation of the concentration dependence of the number of magnetic nanoparticles in the ferrofluid was not reported yet. Fig. 1 (c) shows the difference between GMI responses of FeCoNi composite wire for 100 and 50\% water-based ferrofluids. Various work points (W1, W2, W3, and W4) can be selected for quantitative ferrofluid concentration measurements. It is interesting to mention that $\Delta \mathrm{Z} / \mathrm{Z}$ changes are different for the fields $\mathrm{H} 1$ and $\mathrm{H} 2$ corresponding to GMI maxima of the same branch (Table 1). It might be a consequence of the difference in the magnetization dynamics previously discussed by P. Jantarantana et al [9]. As the next step GMI of the electroplated wire in different regimes in presence of the ferrofluids can be evaluated aiming to study their dynamic properties.

\section{GMI properties of the electroplated wires in the non-uniform field created by the permanent magnet}

GMI curves of the electroplated wires were measured in the condition of the application of the uniform external magnetic field parallel to the wire axis. The highest obtained sensitivity of about $320 \% /$ Oe was observed for a field of about $3.2 \mathrm{Oe}$. GMI responses of $\mathrm{CuBe} / \mathrm{FeCoNi}$ composite wires were also studied in the condition of the application of the non-uniform magnetic field created by $2 \mathrm{~cm}$-diameter/ $1.5 \mathrm{~cm}$-long cylindrical permanent magnet (Fig. 2). The axis of the magnet was perpendicular to the wire axis and cross configuration was symmetrical. In order to evaluate the
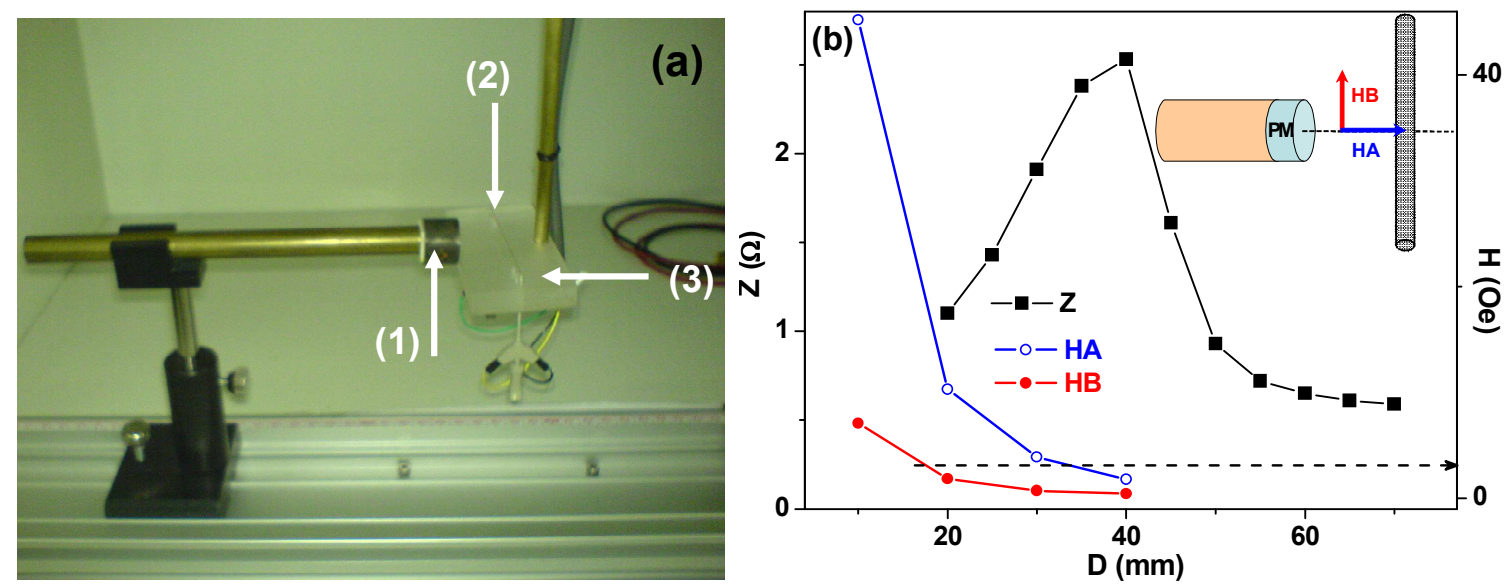

Fig. 2. General view of the GMI measuring system: (1) - Permanent magnet on non-magnetic holder, (2) $\mathrm{CuBe} / \mathrm{FeCoNi}$ electroplated wire, (3) non-magnetic sample holder, (a). Maximum value of the total impedance as a function of the distance, $\mathrm{D}$, between the magnet surface and the wire centre; dependencies of the HA and HB external field components as the functions of the distance from the magnet surface: arrow indicates the value of the external field of 3.2 Oe, corresponding the maximum GMI sensitivity in case of the application of the uniform magnetic field parallel to the wire axis (b). 
values of the magnetic field created by the magnet two magnetic field components (HA and HB, Fig.2 (b)) were measured by the Hall probe for different distances from the surface of the magnet along the magnet axis. Proposed configuration of the wire and the magnet position can be useful for technological applications in the automatic control lines, machinery operations control, rotary heads, and positioning systems [10] where instead of application of the uniform external magnetic field in a big space the local non-uniform configurations can be successfully exploited.

Fig. 2 shows that the maximum value of the impedance correspond to the distances of 35 to 40 $\mathrm{mm}$. There were two intervals of rather high and almost linear sensitivity: for the interval of 20 to $35 \mathrm{~mm}$ the sensitivity of $0.1 \mathrm{Ohm} / \mathrm{mm}$ and for the interval of 40 to $50 \mathrm{~mm}$ the sensitivity of 0.2 $\mathrm{Ohm} / \mathrm{mm}$ was observed. Observed dependence of the total impedance on the distance, $\mathrm{D}$, between the magnet surface and the wire centre can be understood if one takes into account the features of the non-uniform magnetic field created by the magnet. For brief analysis let us consider only the features of the field in the centre of the wire. One can see that to expect, the maximum of $Z(D)$ dependence corresponds to the field interval of the high sensitivity of $\mathrm{Z}(\mathrm{H})$. It is clear from the Fig. 2(b) that the field created by the magnet is significantly non-uniform. It causes the appreciable change in the total impedance of the $\mathrm{CuBe} / \mathrm{FeCoNi}$ electroplated wire up to the distance of about 70 $\mathrm{mm}$ for which the value of all components (HA and HB) become comparable with the local geomagnetic field values. The highest observed sensitivity (for the interval of 40 to $50 \mathrm{~mm}$ ) of 0.2 $\mathrm{Ohm} / \mathrm{mm}$ can be explain if one takes into account non-uniform field distribution over the length of the electroplated wire.

\section{Summary}

Magnetic properties and GMI of $\mathrm{CuBe} / \mathrm{Fe}_{20} \mathrm{Co}_{6} \mathrm{Ni}_{74}$ electroplated wires were studied for frequency of $1 \mathrm{MHz}$ and the amplitude of the driving current of $20 \mathrm{~mA}$ with focus on the development of magnetic field detectors of particular types. The possibility of quantitative ferrofluid concentration measurements was demonstrated. GMI of the electroplated wires in the non-uniform field created by small permanent magnet was studied in the configuration adapted for automatic control lines, machinery operations control, and positioning systems. The maximum of the total impedance absolute value dependence on the distance between the surface of the magnet and the centre of the sensitive element corresponds to the field interval of the high sensitivity of the GMI of $\mathrm{CuBe} / \mathrm{FeCoNi}$ sensitive element.

Support by SAIOTEK MAGNOSEN and ACTIMAT Grants is acknowledged.

\section{References}

[1] B.D. Cullity, Introduction to Magnetic Materials, Addison-Wesley, Reading, MA, USA, 1972.

[2] R.S. Beach, N. Smith, C.L. Platt, F. Jeffers, A.E. Berkowitz, Magneto-impedance effect in NiFe plated wire, Appl. Phys. Lett. 68 (1996) 2753-2755.

[3] G.V. Kurlyandskaya, H. Yakabchuk, E.Kisker, N.G. Bebenin, H. García-Miquel, M. Vazquez, V.O. Vas'kovskiy, Very large magnetoimpedance effect in FeCoNi ferromagnetic tubes with high order magnetic anisotropy, J. Appl. Phys. 90 (2001) 6280-6286.

[4] G.V. Kurlyandskaya, N.G. Bebenin, V.O. Vas'kovsky, Giant magnetic impedance of wires with a thin magnetic coating, Phys. Met. Metall. 111(2) (2011) 133-154.

[5] A.S. Antonov, N.A. Buznikov, A.F. Prokoshin, A.L. Rakhmanov, I.T. Iakubov, A.M. Yakunin, Nonlinear magnetization reversal in Copper-Permalloy composite wires induced by a high frequency current, Technical Physics Letters, 27 (2001) 313-315.

[6] C. Sirisathitkul, P. Jantaratana, Magnetoimpedance of electroplated wires with large core diameters, J. Mater. Sci. Technol. 25 (6), (2009) 825-828.

[7] H. Garcia-Miquel, G.V. Kurlyandskaya, V.I. Levit, Magnetic properties of electroplated wires coated by ferrofluid, J. Magn. Magn. Mater., 300 (2006) e55-e58.

[8] G.V. Kurlyandskaya, M.L. Sanchez, B. Hernando, V.M. Prida, P. Gorria, M. Tejedor, Giantmagnetoimpedance-based sensitive element as a model for biosensors, Appl. Phys. Lett. 82 (2003) 3053-3055.

[9] P. Jantaratana, N.G. Bebenin, G.V. Kurlyandskaya, Magnetoimpedance and magnetization processes of FeCoNi electroplated tubes, J. Appl. Phys. 105 (2009) 013908.

[10] K. Mohri, T. Uchiyama, L.V. Panina, Recent advances of micro magnetic sensors and sensing application, Sens. Act. A 59, (1997) 1-8. 